\title{
SPATIO-TEMPORAL PROCESS SIMULATION OF DAM-BREAK FLOOD BASED ON SPH
}

\author{
Huibing Wang ${ }^{1,2}$, Fanghong Ye ${ }^{1,3, *}$, Sida Ouyang ${ }^{1}$, Zhong Li $^{4}$ \\ 1Satellite Surveying and Mapping Application Center, NASG, China- (wanghb,yefh,ouyangsd)@sasmac.cn \\ 2Jiangsu Center for Collaborative Innovation in Geographical Information Resource Development and Application, China \\ 3School of Resource and Environmental Sciences, Wuhan University, China \\ 4Sichuan Province Second Geographic Information Engineering Institute of Surveying and Mapping, China-mail2lizhong@126.com
}

\section{ICWG III/IVa}

KEYWORDS: Smooth Particle Hydrodynamics, GIS, Dam-break Flood, Spatio-temporal Process Simulation

\begin{abstract}
:
On the basis of introducing the SPH (Smooth Particle Hydrodynamics) simulation method, the key research problems were given solutions in this paper, which ere the spatial scale and temporal scale adapting to the GIS(Geographical Information System) application, the boundary condition equations combined with the underlying surface, and the kernel function and parameters applicable to dam-break flood simulation. In this regards, a calculation method of spatio-temporal process emulation with elaborate particles for dam-break flood was proposed. Moreover the spatio-temporal process was dynamic simulated by using GIS modelling and visualization. The results show that the method gets more information, objectiveness and real situations.
\end{abstract}

\section{INTRODUCTION}

By using GIS modelling method and technologies, the dambreak flood process simulation has become a research hotspot. As one kind of the decision support systems, GIS has the advantages of geographic data management and spatio-temporal analysis, which can substantially provide supports to the spatiotemporal process simulation of dam-break flood.

The traditional GIS normally focuses on simulating analysis using DEM, such as Catchment extraction analysis based on Digital Elevation Model(DEM)(ZHU et al,2005),Surface flow path model integrated from the Terrain mode with the formula of ground water(LIAO et al,2011),flooding analysis method based on regular grids(CHEN et al,2014), the computation of sink streamline and confluence area by aspect and slope of DEM using ArcGIS and hydrological tools, and classifying the risk are by establishing the index model on the basis of various hazards factors(ZHU et al,2009).

In recent years, the microscopic discrete simulation methods have been introduced into GIS spatio-temporal process simulation analysis based on the theory of micro dynamic simulation, such as Cellular Automaton (CA) (YIN et al,2015), neural network(LI et al,2015)and multi-agent(ZHANG et al,2011), etc. These methods can get spatio-temporal logical simulation results of dam-break flood process by fully considering the spatio-temporal relations of various data. However as the physical dynamics factors were ignored, it led to the limitation of the reliability and accuracy of simulation and the lack of 3D realistic and reliable dynamic scene visualization of the spatial-temporal processes simulation. The traditional GIS simulation results are simple and schematic.

One of the approaches to solve the loss of capability to simulate the spatio-temporal processes in GIS environment is integration of GIS and spatio-temporal model (GOODCHILD, et al, 1992) (ZHANG, et al, 2011). Considering from mathematical physics, the research of dam flood can be turned into solving the fluid dynamics equations which control the fluid motion(WANG,et al,2007).

The Smoothed Particle Hydrodynamics (SPH) is a Lagrangian mesh-free particle method, initially appeared in the astrophysical applications of solving complex simulations of planetary motions (LB,1977)(RA et al,1977). At present the $\mathrm{SPH}$ is well applied on simulating general fluid dynamics issues, and mainly used in fluid mechanics and related fields (LIU et al,2003). Regarding the simulation of dam flood process, SPH has two advantages. On one hand, the SPH focus on the principle and laws of internal geographic spatio-temporal processes. The geographical objects are decomposed into innumerable particles, and the movements of each particle have been simulated. Thus, the simulation results reveal the inner essence and regularity of the changes of geographical spatialtemporal process. On the other hand, benefiting from the SPH with the characteristics of Lagrangian mesh-free, convergence proprieties of compacted domain and particles discreteness, it is easier to get the numerical solution of fluid control equations.

Considering the principle of dynamics of the spatio-temporal dam break, the integration of algorithm model was adapted to the application of GIS in this paper, proposing the approach and computational method of the spatio-temporal process of dam break flood. With the outcomes of simulation data of time series of the spatio-temporal evolution and changing process, the expression of dynamic visualization was implemented using GIS technologies. Therefore, the problems of subjectivity and lacking sense of real scenes and situation of dam-break flood simulation in traditional GIS system were solved. Moreover, a decision-making approach and toll was provided for flood disaster prevention and reduction. 


\section{THEORIES}

\subsection{Analysis of Dam-break Flood Spatio-temporal Process}

In the applications of GIS, the simulation of dam-break flood spatio-temporal process can be used to foresee and prepare the emergency plan for the risks of the dam failure, or to restore the process of dam break which has occurred. In terms of Math Physics field, in this paper, a hydrodynamic process of fluid motion in 3D spatial scene was described for the dam- break flood spatio-temporal process, which was caused by water flowing downstream due to gravity after the dam-break, the internal forces include pressure and viscous forces in the water body, as well as the common action of the external force, such as the friction force and the reaction force generated by the water body.

In this regards, the simulation of dam break flood spatiotemporal process is decomposed into following several levels in this paper, including

1. Selecting fluid control equations to describe the process of dam break and to abstract actual dam break process into dynamic equations.

2. Using SPH method to resolve the dynamic equation of dam flood break into numerical calculation formula. Moreover, the numerical simulation results of dam-break flood can be obtained by calculation.

3. Using GIS modelling and visualization methods to introduce numerical simulation results as GIS data, and to realize the evolution and visual analysis of the dam-break flood spatiotemporal process.

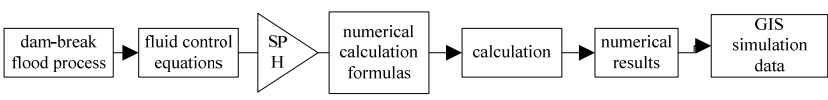

Fig.1 The flow of simulation method for dam-break flood spatio-temporal process

\subsection{Fluid Control Equation of Dam- break Flood}

The dynamic characteristics of fluid motion are described by the fluid control equations. According to the characteristics of dam-break flood which can be regarded as the viscous surfacefree fluid, the Navier-Stokes(NS)equations are selected as the fluid control equations of the dam-break flood in this paper.

One of NS equations, partial differential expression of Newton's second law in viscous fluid, is generally described as follows (GK, 1967),

$$
-\bar{p}+\bar{F} \cdot T+f=p \frac{\mathbb{D v}}{D t}
$$

The right side of the equation is the total momentum of the infinitesimal fluid body element. $P$ is the density which can be regarded as the mass $\mathrm{m}$ of infinitesimal fluid body element, where $\frac{}{\mathrm{DV}}$ is the acceleration.

The left side of the equation is the force acting on the infinitesimal fluid body element, which is composed of surface force and volume force. $\nabla$ is Hamiltonian operator, $\mathrm{P}$ is pressure, and $\mathrm{T}_{\text {is viscous stress tensor. While }} \mathrm{f}_{\text {is }}$ the volume force, that is non contact force, such as gravity, electromagnetic force, and inertia force, etc.

\subsection{Solutions of SPH}

The basic principle of SPH is to obtain the mechanical behaviour of the whole system by solving the dynamic equation of the particle group and tracking the trajectory of each particle, with the continuous fluid (or solid) described by using an interacting particle group, and each particle carrying a variety of physical quantity, including quality, speed, etc(Ma, et $\mathrm{al}, 2008)$. The problem domain of SPH is described by a particle which carries the coordinates of the elements of the space and the physical field rather than a grid, and the finite particles are viewed as approximate computational framework of the physical field .The physical field function is calculated by the smooth kernel function integral approximation in the compact support domain.

\subsubsection{Integral Approximation}

According to the definition of Dirac's function, the integral expression of any function is described as the followings.

$$
f(x)=\int_{-\infty}^{1-} f\left(x^{\prime}\right) \delta\left(x-x^{\prime}\right) d x^{\prime}
$$

Smoothed kernel function whose character is similar to Dirac's function is defined in SPH to replace the Dirac's function, with the integral approximate expression as followings.

$$
f(x) \approx \int_{-k h}^{+k h} f\left(x^{\prime}\right) W\left(x-x^{\prime}, h\right) d x^{\prime}
$$

Where, $\mathrm{h}$ is the smooth length, to define the compactly supported domain of the smoothed kernel function. While the $[-\mathrm{kh},+\mathrm{kh}]$ is compactly supported domain.

\subsubsection{Particle Approximation}

Regarding the continuous space field, the SPH method can be described by the discrete particle distribution.

The physical quantity $\mathrm{u}\left(\vec{x}_{\mathrm{i}}\right)$ of the particle $\mathrm{i}$ on the space position $\vec{x}_{\mathrm{i}}$ can be approximated by the weighted sum of the physical quantities $\mathrm{u}\left(\bar{x}_{\mathrm{j}}\right)$ of all the particles $\mathrm{j}$ in the compactly supported domain of particle ${ }^{\mathrm{i}}$. Thus, the function of particle approximation is described as followings.

$$
\mathrm{u}\left(\vec{x}_{\mathrm{i}}\right) \approx \sum_{\mathrm{j}=1}^{\mathrm{N}} \mathrm{u}\left(\vec{x}_{\mathrm{j}}\right) \mathrm{Q}\left(\vec{x}_{\mathrm{j}}\right)
$$

Where, $\mathrm{Q}\left(\vec{x}_{\mathrm{j}}\right)$ is the weight function at the spatial position.

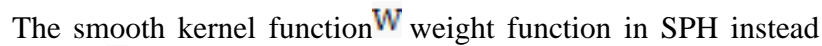
of $Q$,which is expressed by the calculation formula of the infinitesimal meta volume, that is ,mass divided by density $\frac{\bar{m}}{\bar{p}}$, then the final expression for the SHP solution of the physical equation $f\left(\vec{x}_{\mathrm{i}}\right)$ is obtained as follows.

$$
f\left(\vec{x}_{\mathrm{i}}\right) \approx \int_{-\mathrm{kh}}^{+\mathrm{kh}} f\left(\vec{x}_{\mathrm{j}}\right) \mathrm{W}\left(\vec{x}_{\mathrm{i}}-\vec{x}_{\mathrm{j}}, \mathrm{h}\right) \mathrm{d} \vec{x}_{\mathrm{j}} \approx \sum_{\mathrm{j}=1}^{\mathrm{N}} f\left(\vec{x}_{\mathrm{j}}\right) \mathrm{w}_{\mathrm{ij}} \frac{\mathrm{m}_{1}}{\hat{p}_{1}}
$$

where $\mathrm{w}_{\mathrm{ij}}=\mathrm{W}\left(x_{\mathrm{i}}-x_{\mathrm{j}}, \mathrm{h}\right)$ 


\subsection{Computation Procedure of Simulation}

Using the SPH method to solve the fluid control equations the simulation computation process of dam-break flood can be realized by the following steps.

At a certain time point $t_{0}$, the coordinates of the infinitesimal fluid body element in fluid can be set up as $x_{0} 6 X_{0}, Y_{0}, z_{0}$ t , with a speed of $v_{0}\left(v_{x_{0}}, v_{v_{0}}, v_{v_{0}}\right)$ and the density $\rho_{0}$ at the current time. Then first the final expression for the SHP solution known as formula(5) will be used to sum up some integral of the physical quantity of the particles in a compactly supported domain, such as the rate of density change, the pressure acceleration, the viscous force acceleration, the volume force acceleration, etc. Secondly, the calculating the speed of the next time point, which is shown as $p_{1}$, as well as the pressure term on the left side of the equation(1) is needed. Thirdly, the stress tensor term and the volume force term in order to get the speed change rate on the right side of the equation, so as to obtain the speed of the next time point which is expressed as $v_{1}$ is required to the calculation of the position of the infinitesimal fluid body element at the next point in time with the expression of $x_{1}\left(X_{1}, Y_{1}, z_{1}\right)$.The spatial distribution of all the infinitesimal body elements can represent the spatial distribution of the whole dam-break flood, and the space state of the dam-break flood at all times can express the complete spatio-temporal process of dam-break flood. The flow of the calculation process of simulation based on SPH concludes of the above steps is shown at below,

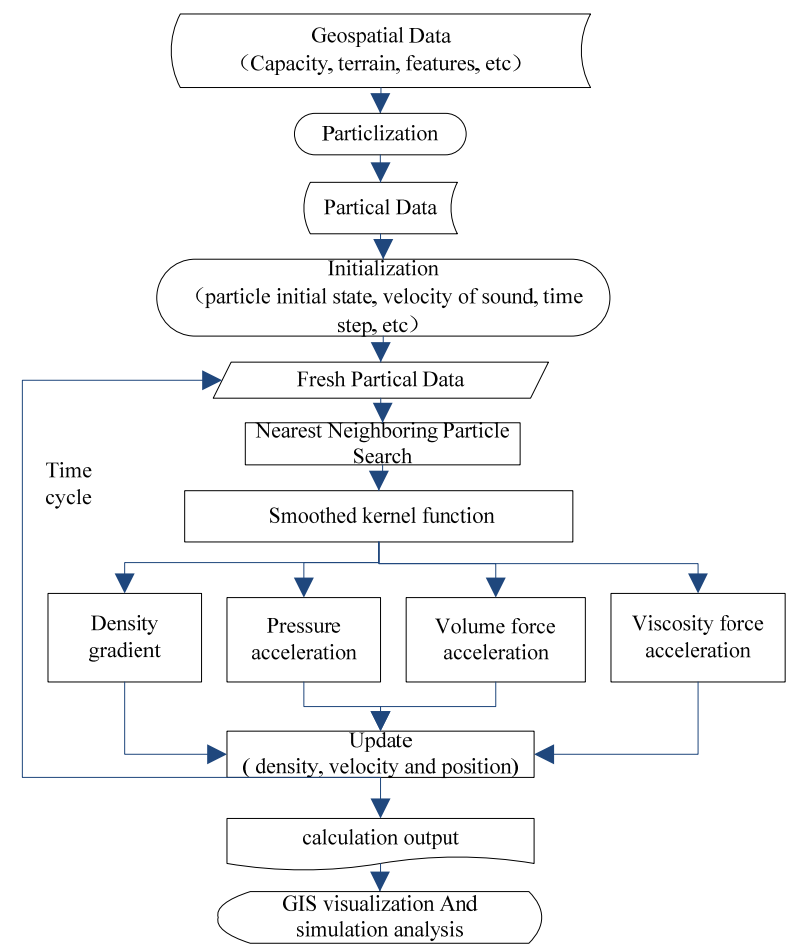

Figure 2. The flow of simulation calculation based on SPH

\section{KEY TECHNOLOGIES}

\subsection{Spatial Scale}

3D volume models are represented by Surface patch grid in GIS systems. However describing $3 \mathrm{D}$ solid objects in the form of particles is the more effective way for the spatial data in SPH simulations and computations. Therefore the geospatial data is particle-ized as the inputs to the simulation and the computation.

During the particle-ization, the shape of solid object cannot be presented accurately if the size and diameter known as $\mathrm{d}$ of particle is too big. On the contrary, when the diameter of the particles is too short, which leads to the large number of the particles will affect the efficiency of the computation.

In the application of dam break flood simulations of this paper, the particles were set up with the diameters of from 5 to 10 meters, with the number of particles reaching hundreds of thousands to millions after the particle-ization.

Moreover, another key parameter influencing the spatial scale of the simulating and computational system $\mathrm{m}$ is the local speed of sound expressed by c, which limits the maximum space displacement in arbitrary systems.

According to the definition of Mach number, the flood can be assumed as compressible flow when $M=\frac{\mathrm{v}}{\mathrm{c}} \leq 0.3$.

In the simulation of dam break flood, the compressible property of the dam break flood is not able to be simulated, if $\mathrm{C}$ adopted by the actual sound velocity, due to the small value of Mach number. Therefore, the local speed of sound expressed by $\mathrm{c}_{\text {is }}$ necessary to be introduced in, based on various spatial scales of simulated scenes. The relations of the local speed of sound and the spatial scale are defined as the followings,

$$
\mathrm{c} \leq \frac{\sqrt{2 \mathrm{~g} \Delta \mathrm{H}+v_{0}^{2}}}{0.3}
$$

In the formula, $\mathrm{g}$ is Acceleration of gravity, $\Delta \mathrm{H}$ is spatial height difference, and $v_{0}$ is the overall initial velocity of the fluid.

In the research of this paper, Dam break flood starts from the dam break ,the overall initial velocity of the fluid were taken as the value of zero,and ${ }^{\mathrm{C}}$ were taken the maxi value to fully achieve the proximity of the real fluid, which is shown as the following,

$$
\mathrm{s}-\frac{\sqrt{2 g S M}}{0.6}
$$

\subsection{Temporal Scale}

The physical parameters can be calculated by solving the fluid dynamics equations which control the fluid motion. Driven by time iteration, using the particle physical quantities which are continuously updated at the current point in time to calculate the next point in timeline, can realize the estimation of the whole simulation process at any time point.

The time resolution depends on time step of iteration represented by $\boldsymbol{\Delta}$, as well as $\Delta$ also affects the efficiency and accuracy of the simulation. The simulation results maybe physically wrong if the state parameters of particles can't be updated timely caused by long time steps. If the value of $\Delta t$ is too small ,the value of results of every iteration may have little 
changes to be detected, and the calculation efficiency is decreased.

To avoid the Non -physicalphenomena, considering the Courant-Friedrichs-Lewy condition, the dependent region of numerical solution should include all possible solutions. In the SPH method, Courant-Friedrichs-Lewy condition is the compacted $[-k h,+k h]$ as the support domain being greater than the maximum possible displacement of at ,which can be presented as the following,

$$
\text { st }<\frac{2 \mathrm{kh}}{6}
$$

\subsection{Equations of Boundary Conditions}

In the process of dam-break flood simulation if the body force suffered by the fluid only considers gravity and ignores the support or obstruction from objects on underlying surface, the particles would pass through the underlying surface and cause wrong physically results. Boundary force model for the geospatial data of underlining surface was necessary to be added to boundary force worked on boundary fluid particles as additional body force in the description of fluid dynamics control equations. Integrated with the properties of topography and feature as well as the spatial scales, boundary condition equations were built to simulate the effect of reaction force to fluid particles generated from the underlying surface, buy using the boundary force $F_{\mathbf{b}}$ as the Additional volume force.

In this paper, the calculation of boundary force used the similar Lennard-Jones formulas on calculating molecular force ,where boundary particles only exert force which is $\mathbb{F}_{b}$, along the central line of fluid particles in its affected range, formulized as the following.

$$
\frac{\mathrm{D}_{\mathrm{F}_{n}}}{\mathrm{Dt}}=\left\{\begin{array}{cc}
0.01 \mathrm{~s}^{2}\left[\left(\frac{l}{r_{n}}\right)^{12}-\left(\frac{l}{r_{n}}\right)^{4}\right] \frac{1}{r_{\eta}}, & \left(\frac{l}{r_{n}}\right)<1 \\
0, & \left(\frac{l}{r_{n}}\right) \geqslant 1
\end{array}\right.
$$

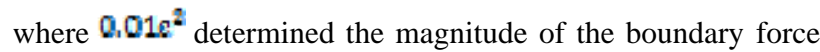
which relates to the spatial scale. While $r_{i j}$ is the distance between the boundary particle and the other solid particles, and the boundary force grew in inverse proportion to the distance of the particles. $l$ was the influence radius of the boundary particles, when it grew, the influencing range of the boundary particles increased and the kinetic energy loss of a fluid particle became more. Thus, the different sizes of $l$ was able to be used for simulating the resistance influence on fluid of different terrain and features with different underlying surfaces.

\subsection{Smoothed Kernel Function}

Smoothed kernel function $W$ is the key to determine the applicability and the precision of the solutions in SPH method and $W$ can be any constructor with the similar properties of Dirac's function. In this research of dam-break flood simulation, fifth order spline function was selected and, which was more stable to irregular distribution of the particles and was near to full smoothness (JP,1996).

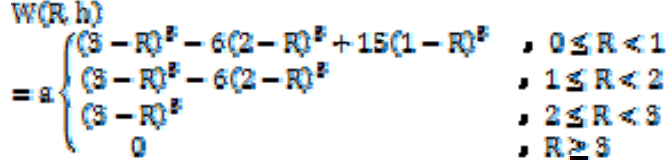

where, $\mathbb{R}=\frac{\left|\hat{A}-z_{1}\right|}{\mathrm{h}}$, and in the three-dimensional coordinated space, ${ }^{\mathrm{a}}=\frac{\mathrm{g}}{\mathrm{g} \text { anb? }}$.

The interpolation length of smooth kernel, which is $\mathbf{h}$ and the radius of the compacted support domain, which is th directly affected the calculation efficiency and precision of the solution.(LIU et al,2003).Small value of interpolation length may cause the problems like numerical fracture, while the huge value of interpolation length can lead to the sharp increase for amount of computation, with the affections to the local precision(JW, et al,1994)

The simulation of dam break flood in the paper proposed the comparison of the computing experiment by selecting interpolation length of $\mathbf{h}$ as different multiples of different particle size determined by $d$.
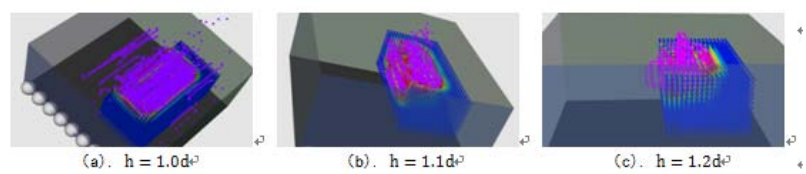

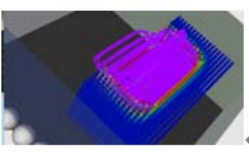

(d). $h=1.3 \mathrm{~d}^{2}$

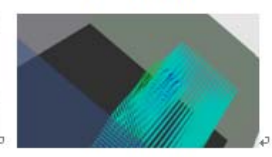

(e). $h=1.4 \mathrm{~d}^{3}$

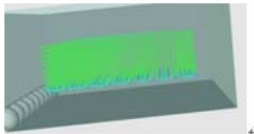

(g). $h=1.6 \mathrm{~d}$

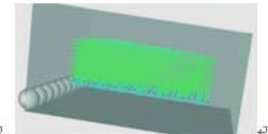

(h). $h=1.8 \mathrm{~d}$ (f). $h=1.5 d$

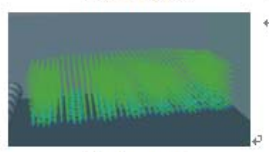

(i). $h=2.0 \mathrm{~d}^{3}$

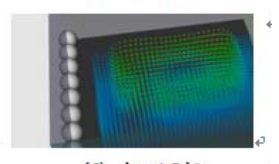

Figure 3. The comparison of different calculation results using different smoothing length

The figure shows the splash phenomenon caused by the small interpolation length (i.e., when $h<1,2 \mathrm{~d}$ ), and the loss of local dynamic features on the peak of the flood when the interpolation length was large(i.e., when $h>1.5 \hbar$ ). Therefore, reasonable interpolation length in simulation of dam-break flood was selected by the adoption of,

$$
1,2 \mathrm{~d}<\mathrm{k}, 51,2 \mathrm{k}
$$

Considering the computation level, the compact support domain radius th was defined 2 times as the smooth length. In the three-dimensional space, the order of magnitude in each particle compact support domain was limited to around 100.

\subsection{Particle Modelling}

The basics of achieving the 3D dynamic visualization and analysis of he dam-break flooding evolution is the effective organization of enormous fluid particles produced by simulation of the process of dam-break flooding using SPH method. Constructing surface grid of fluid particles during each time 
tense can greatly reduced the fluid data volume, as the internal particles have little influence to visualization and analysis. Therefore this paper conducted the simulation procedure of the fluid particles as the steps followed,

1.Constructing a regular three-dimensional grid volume data set based on scattered fluid particle data, and assigning each vertex in three-dimensional grid a scalar value by Gauss distribution function.

2.Extracting surface grid data by Marching Cubes method(Lorensen et al.,1987) from the data set based on step1.

3.Compressing and smoothing the surface grid data using edge collapse algorithm(Hoppe et al, 1993) and Laplasse smoothing algorithm(Sorkine et al.,2004) to imporve the rough and enormous triangular patch produced by Marching Cubes method as needed.

Through the modelling process as the aboves, the fluid surface gird data of fluid particles at each tense was converted and illustrate in Figure 4.

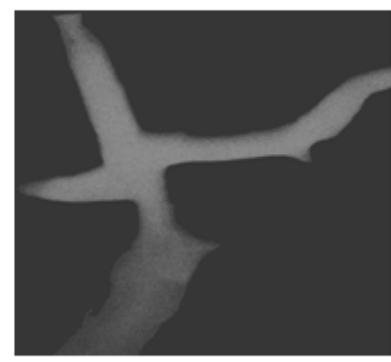

a. Fluid Particle

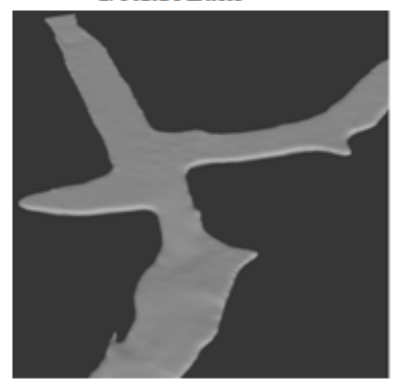

c. Compression and Smoothing

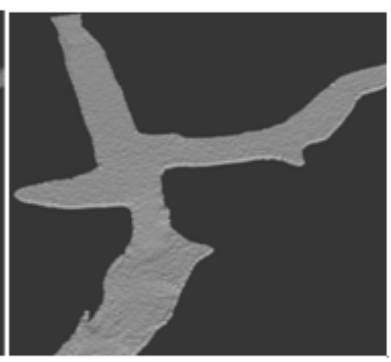

b. Fluid Surface Mesh

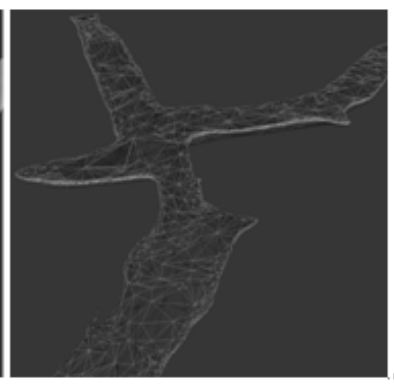

d. Triangular Mesh Display
Figure 4. Fluid Particle Modelling

\section{ANYLYSIS OF A CASE STUDY}

A medium-sized reservoir of 80 million cubic meters of storage capacity was selected as an example in this paper, to simulate the submerged condition of dam-break flood on the reservoir area, as well as on the downstream area with the terrain range of 100 square kilometres.

Based on the voxel-oriented method of 3D mesh model divided by the octree structure(WU et al,2004), the particle-ization was implemented to the geospatial data, such as flood body, land surface and land features,etc.600,000 particles of water body and 5 million of boundary particles was created as the inputs of the simulation and computation, with the scale of 5 meters set up for each particle. The result of the particle-ization was shown in Figure 5.

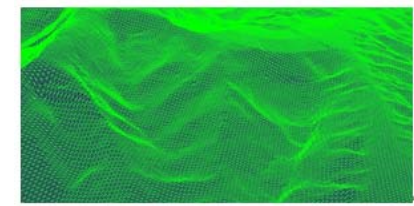

a. Topographic surface triangular mesh model

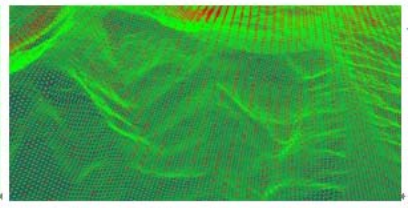

b. Terrain model particle-ized
Fig.5 The surface particle-ization

In a Multi-core parallel computing environment and using high performance graphics workstation (8 core 2.6GHzCPU, 32G memory), the simulation of dam-break flood process which supposed to be evolved in 1000 seconds took about 10 minutes to complete, outputting 1000 files of the status information of the fluid particles. Each file contained the status information of each particle in the tense at present, which included the serial numbesrs, coordinates and various physical properties, such as the mass, density, pressure, velocity, and the acceleration, etc.

The visualized analysis of dam-break flood spatio-temporal process was achieved by generating the surface mesh data of the particle swarm establishing the 3D surface model of the fluid in each tense, and using the 3D dynamic visualization technology of spatio-temporal data (WANG et al, 2013).
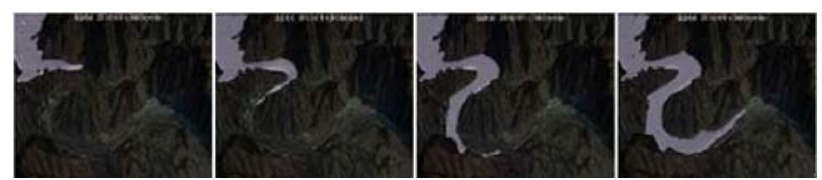

Figure 6. The dynamic visualization of a dam-break flood spatio-temporal process

Compared with the traditional simulation using DEM, the characteristics of dam-break flood process simulation based on SPH method are analyzed. The simulation based on SPH required the huge computation, but outputs of the computation regarding the spatio-temporal data existed with very rich information, which contains not only the distribution information of dam-break flood at each moment, but also the abundant hydrological monitoring information in each spatial location, such as water velocity, density, water level, etc. In particular, the changes of positions and properties of the particles could be analyzed and tracked. Meanwhile, as the calculation formula of dam-break flood simulation is derived from the principle of fluid dynamics, the simulation results indicated that the physical process of flood flow evolution became more objective and realistic. Due to the analyzability of the disaster scenarios provided by the spatio-temporal process of the dam-break flood simulation results, it is suggested to integrate with the vector data of the features of the basin area to get the statistics in real time for the flooding area and villages to conduct the disaster process analysis. The spatio-temporal process of dam-break flood realized by simulation has analyzability of the disaster scenario in space and time.

For example, at the 14th minute after the dam-break happened, the flooding inundated area had reached 8,351,435.77 square meters with 8 villages submerged, shown in figure 7. 


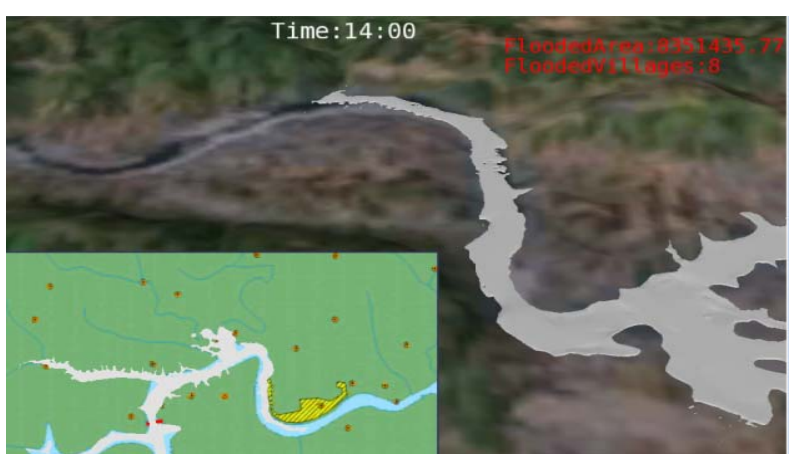

Figure 7. The analysis on dam-break flood evolution process

\section{CONCLUSION}

Large number of reservoirs existed in China, which might have great potential risk of dam break causing serious flood disaster. The simulation analysis and prediction evaluation of dam break have always been the important scientific methods of disaster prevention and reduction over the world. This paper proposed the simulation and computation method by using fine particles of dam-break flood geospatial process based on SPH, adopting the high-performance computing technologies to generate spatio-temporal sequences simulation data which is introduced to Geographic Information System as the innovated dynamic data sources. The multi-level 3D dynamic scenarios based on the integration of geospatial data and simulation data were constructed successfully to demonstrate the evolution process of the dam-break flooding using the dynamic visualization method. Moreover

the statistical Analysis of synchronous disaster effects was able to be conducted synchronously to achieve the scenario analysis of the dynamic visualization.

This innovated method on SPH can provide rich spatialtemporal process data to support warning and prevention plan, plan of infrastructure, post-disaster restructure analysis and decision making of disaster rescue of the dam break disasters. Definitely, the method of simulation of spatial and temporal process based on SPH in this paper can also be adapted to the evolution simulation analysis of typical disaster processes, such as debris flows, storm surges, landslides, etc. Nevertheless, the limitations of this method occurred, such as huge quantity of calculation and time consuming. Thus, to increase the efficiency of this method, further research and improvement is required to be promoted for the parallelization of the computation processes.

\section{REFERENCES}

CHEN Jing, YUAN Sijia, ZENG Fangmie. 2014. Virtual Globe-based Source Flood Submergence Analysis Algorithm. Wuhan Daxue Xuebao, 39(4):492-495+504.

GK Batchelor, 1967.An Introduction to Fluid Dynamics. Cambridge: Cambridge Universitv Press.

Hoppe H, DeRose T, Duchamp T, et al., 1993. Mesh optimization/Proceedings of the 20th annual conference on Computer graphics and interactive techniques. ACM, pp.19-26.

JP Morris,1996. Analysis of Smoothed Particle Hydrodynamics with Applications. Monash University,Australia.
JW Swegle, SW Attaway, MW Heinstein, et al., 1994.An Analysis of Smoothed Particle Hydrodynamics. Nasa Sti/recon Technical Report N,DOI:10.2172/10159839

LB Lucy, 1977.A Numerical Approach to the Testing of the Fission Hypothesis. Astronomical Journal,82(82):1013-1024.

LI Weihong, CHEN Yebin, WEN Lei,2015.Simulation of Spatio-temporal Diffusion of Dengue Fever Based on the GABP Neural Network Model. Journal of Image and Graphics, 20(7):981-991.

LIAO Yongfeng, NIE Chengjing, 2011. A Disaster Risk Warning Model for the Basin-wide Rainstorm Flood Management. Journal of Geo-Information Science, 13(3):354360.

LIU Guirong, LIU Moubin,2003.Smoothed Particle Hydrodynamics: A Meshfree Particle Method. World Scientific.

Lorensen W E, Cline H E, 1987. Marching cubes: A high resolution 3D surface construction algorithm/ ACM SIGGRAPH computer graphics. ACM, 21(4),pp 163-169.

MA li, BAO Ronghao, GUO Yimu, 2008.Waterjet Penetration Simulation by Hybrid Code of SPH and FEA. International Journal of Impact Engineering, 35(9),pp, 1035-1042.

GOODCHILD Michael, ROBERT Haining, 1992.Integrating GIS and spatial data analysis:problems and possibilities. Geographical Information Systems, 6(6):407-423.

RA Gingold, JJ Monaghan, 1977. Smoothed Particle Hydrodynamics - Theory and Application to Non-spherical Stars. Monthly Notices of the Royal Astronomical Society,181(2),pp,375-389.

Sorkine O, Cohen-Or D, Lipman Y, et al, 2004. Laplacian surface editing[C]/ Proceedings of the 2004 Eurographics/ACM SIGGRAPH symposium on Geometry processing. ACM, pp, 175-184.

WANG Huibing, TANG Xinming, OUYANG Sida, et al, 2013.Target and Implementation of Dynamic Visualization of Geographic Spatio-temporal Process. Science of Surveying \& Mapping, 38(6),pp,85-87.

WANG Lihui, HU Siyi, 2007.Study on Dam Failure-related Problems[J]. Advances in Science \& Technology of Water Resources, 27(1),pp,80-85.

WU Xiaojun, LIU Weijun, WANG Tianran, 2005.Octree Structure Based Voxelization of Polygonal Meshes. Journal of Engineering Graphics ,26(4),pp,1-7.

YIN Lingzhi, ZHU Jun, WANG Jinhong, et al, 2015.Real-time Simulation and Analysis of Dam-break Flood Routing Based on GPU-CA Model. Wuhan Daxue Xuebao, 40(8),pp,1123-1129.

ZHANG Honghui, ZENG Yongnian, TAN Rong, 2011.A Spatio-temporal Simulation and Planning Model for Farmland Conversion Based on Multi-agent Systems. Acta Geodaetica Et Cartographica Sinica, 40(3), pp,366-372.

ZHANG Xianfeng, CUI Weihong, 2001.Integrating GIS with Cellular Automaton Model to Establish a New Approach for 
Spatio-temporal Process Simulation and Prediction. Acta Geodaetica Et Cartographic Sinica, 30(2),pp,148-155.

ZHU Hongying, GU Huaqi, GUI Xin, et al, 2009.Analysis, Simulation and Visualization of Flood Inundation Based on ArcGIS. Bulletin of Surveying \& Mapping, (5),pp,66-68.

ZHU Qing, TIAN Yixiang, ZHANG Yeting, 2005.The Extraction of Catchment and Subcatchment from Regular Grid DEMs. Acta Geodaetica Et Cartographica Sinica, 34(2),pp,129-133. 